\title{
A Collaborative Science Learning Game Environment for Informal Science Education: DinoQuest Online
}

\author{
Walt Scacchi and Robert Nideffer \\ Game Culture and Technology Laboratory \\ University of California, Irvine \\ Irvine, CA 92697 \\ \{wscacchi, nideffer\}@uci.edu \\ Joe Adams \\ Discovery Science Center \\ Santa Ana, CA \\ 2500 N. Main St. Santa Ana, CA \\ 714-542-2823
}

\begin{abstract}
We describe concepts and results that arose from the development and deployment of a large-scale collaborative game environment called DinoQuest Online. DQO provides an entertaining experience and approach to informal science education. DQO represents a collection of 13 games for helping school-age children to learn about science (or more specifically, life science and dinosaurs). In this paper, we identify and examine different collaborative group forms that emerged to play DQO. Along the way we provided examples of the collaborative groups and game play from DQO.
\end{abstract}

\section{Introduction}

We have developed a large-scale collaborative game environment deployed in a regional science center that joins physical and online activities in the domain of informal science education. The Discovery Science Center (DSC), located in Santa Ana, $\mathrm{CA}^{1}$, is a regional science center that families and school groups visit in order to experience a diverse variety of interactive science exhibits. These exhibits bring scientific subjects or concepts to life in a hands-on, fun, and entertaining manner. DSC focuses on interactive exhibits as opposed to passive exhibits of scientific artifacts as might be found in a museum that memorializes the history of scientific concepts, scientists, and inventions. DSC is also situated in municipal region, Orange County, CA, whose population spans large concentrations of ethnic immigrants (from Mexico and Latin America, Asia, Middle East, etc.), as well as very affluent to very poor communities. During 2005, more than 275,000 people engaged in DSC visits or outreach activities, while in 2007 more than 425,000 people were engaged, including $150,000 \mathrm{~K}-12$ students of which nearly 90,000 participated in school group visits to DSC. Thus, DSC [2008] exhibits and educational outreach programs are tailored to meet the interests of different communities, age groups, school educators, and other constituencies.

\footnotetext{
${ }^{1}$ See, for example, http://www.answers.com/topic/santa-ana?cat=travel
} 
In 2004, effort began at DSC to develop a new interactive game-based exhibit that would focus on dinosaurs as the basis for introducing, demonstrating, and engaging visitors with the concepts from life science (e.g., skeletal systems, elements, and function; digestive system; prey-predator relationships). The life science concepts selected for presentation in the exhibit were those that correspond to curricular topics found in K-6 grade science education standards for California, which are nearly identical to the National Science Education Standards ${ }^{2}$. The UCI Game Culture and Technology Laboratory [UCGameLab 2008] was invited to join this project at this time, and project went into design and operational planning in early 2005. This exhibit was designed to enable the development and deployment of both a physical game-based interactive exhibit at the DSC that would be linked and integrated with a Web-based online game environment. The physical exhibit called DinoQuest (DQ) (see Figure 1) became operational in mid 2006, while the online game environment called DinoQuest Online (DQO), went into full-scale operation in 2007. Both DQ and DQO were conceived, designed, and deployed as collaborative science learning game (SLG) environments, and can be evaluated as such. The remainder of this paper focuses on examining and explaining DQO as an entertaining and collaborative SLG environment, as well as what facilitates different forms of collaboration and collaborative game play in DQO. A companion paper further explores collaboration forms and affordances found in DQ and DQO, individually and collectively [Scacchi, Nideffer, and Adams 2008].

\section{Informal Science Education through Science Learning Games}

Informal Science Education (ISE) is concerned with providing and experiencing scientific concepts, methods, and devices drawn from different science disciplines in settings outside of school, where formal science education occurs. Science centers, museums, after school clubs, and public media (e.g., the Nova television series broadcast in the U.S over the Public Broadcasting System/PBS) are the common settings for ISE, though ISE can also occur at home in settings with family or friends. What is key to ISE is that it is elective, discretionary, and a matter of free choice in terms of the content provider, as opposed to schools whose choices may be determined by school boards or others. However, in our view, science centers that showcase interactive, hands-on exhibits are an ideal setting to deploy SLGs, as part of an overall environment for ISE that is readily accessible to a large public audience.

\footnotetext{
${ }^{2}$ http://www.nap.edu/readingroom/books/nses/overview.html. These standards are not part of recent U.S. Federal initiatives like "No Child Left Behind" nor are they the basis for testing scientific knowledge by school grade. Instead, they focus on identifying for teachers, parents, and others what scientific concepts and practices students should be taught and learned (hopefully) in order to become scientifically literate citizens through their K-12 education. Students who excel or become enthusiastic learners of such materials may then be prepared for college level study and a career in a science, technology, engineering, or mathematical field.
} 
SLGs are a small and mostly marginalized genre of computer games when one looks at the international computer game industry. No companies appear to be making millions of dollars from their best-selling SLGs. In fact, most of the large, well-known computer game companies avoid developing games that are envisioned as "educational" and targeted to specific age-skill groups. Instead, they more often seek to develop games that are fun, entertaining, and engaging, as well as focused on fantasy worlds, rather than on education and academic subjects. Subsequently, there is comparatively little industry interest in developing and deploying educational games in general, and SLGs in particular. However, as some game scholars and educational theorists have observed, many computer games succeed because they are great learning environments that embody both classic and modern theories of constructivist learning, self-identity through role play, reflective thinking, domain-specific specialist language skills, and multi-player socialization [Bainbridge 2007, Gee 2003, Shaffer 2006].

\section{DinoQuest Online}

The DinoQuest Online venue-The DQO venue is a publicly available Web site that downloads a Flash-based DQO game engine needed to play the game. The DQO game engine dynamically loads the content associated with each game module. DQO currently supports 13 game modules. Each module is a game, and the modules are partially ordered and game play results/knowledge are accumulative. The DQO game modules provide a set of simulated environment, some literal, other strictly conceptual. Figure 2 provides a view of the in-game home for DQO that appears as a multi-media computing laboratory or collaboratory [cf. Collabs 2008, Teasley and Wolensky 2001]. In the figure, the large multi-panel wall display serves as the in-game interface for "connecting" to remote collaboratories in geographic locations. Selecting one of these collaboratory panels transitions the user to the associated game modules. The large map display is the "DinoSphere" which is a higher level, multi-player game space that is accessible only after completing the other game modules. The doors to the right take the user to "MyLab", which is the user's private in-game laboratory office where their research points and other objects collected through DQO are kept for later use. The computer screen in the foreground is an interface to an embedded multi-media presentation from an avatar. Most of the 13 game modules can be played by a single user, but based on observations at the DSC, children often play DQO with an adult/parent companion who wants to share the game play experience, engage the child player with a discussion about game play, or seek an explanation from the child about what's going on in the game (more often than not, the child needs to explain how the game works to the parent/adult who doesn't usually play computer games).

DQO game environment-DQO represents a contemporary game platform. It is coded in Flash 8, which runs in most commonly available Web browsers (e.g., Internet Explorer, Firefox, Safari). It is accessed from a single Web site (www.DQOnline.org), which in turn downloads the DQO game engine into the 
user's Web browser, which in turn downloads each game module and its content on user demand. Many families and school groups access and run the DQO game environment on a desktop or laptop computer, as DQO does not require high-end microprocessors, graphics accelerator cards, or the like. Accordingly, the design of the DQO game environment was conceived to enable the largest possible audience of end users or players, including those who may have older, less powerful computers, which includes many under-privileged schools.

Multi-genre game design-As previously indicated, DQO consists of 13 game modules. Collectively, they take a player about 3-5 hours to play to completion. However, individual game modules vary in the duration, exposition of life science concepts, and game genre. For example, DQO game modules includes games drawn from quest, design/simulation, puzzle, and mini-game genres. However, it may also be fair to say that these games can individually or collectively be viewed as "casual games" that can be started, played for a brief period, stopped, and restarted later. However, game scores and research points earned persist across game play sessions, as long as the same user (identifier) is playing.

Embedded multi-media content-DQO incorporates multi-media assets originally created for presentation at the DSC. Figure 4 shows an example of an embedded video file that is played on command, and provides a brief explanation of the goals and levels found in DQO. Use of these in-game characters DQO enables a dual coding of the role models with SLG constructs [Rieber 2005] and creates a sense of continuity in content and play experience in an online environment.

Embedded tutorials for teachers and parents - Gee [2003] reports that children who learn to play games in such a manner often acquire deep knowledge of the ingame specialist language, terminology, and game play moves that are difficult to determine by a competent adult just by reading a game manual. However, in order to help satisfy the requests from parents, teachers, and other educators, we added a series of embedded tutorials and in-context explanations to help teachers and parents better understand what their children may already know. Figure 5 provides a display of in-game help that is part of such a tutorial for one of the game modules. Beyond this, as DQO players progress from game module to module, DQO also displays interstitial (and stylized) text panels that provide further contextual information about some of the underlying scientific concepts or discoveries that are recreated in the game. These interstitial images (or cut scenes) also serve to occupy the player group (e.g., child and parent) with a simple diversion while the next game module in being downloaded and readied for play.

Contemporary game play practices used to elucidate life science concepts-SLGs, as games, need to be more than just interactive presentation of scientific concepts, or simulations of scientific practices or processes. As such, we sought to find way to utilize both original and familiar game play mechanisms and play practices in 
developing each of the DQO game modules. For example, in Figure 6, we see a view of the ecological relationships game module within DQO that enables play with prey-predator and food chain relationships. This module utilizes a "Tetris" style of game play, where a configuration of ecological elements (carnivores/predators, herbivores/prey, and plants) can be rotated as they move from left to right to match up with configurations that have already been anchored, in order to maximize the matches (e.g., carnivores prey on herbivores, herbivores prey on plants, unmatched carnivores die and help nuture plants). As such, we (and many adult players familiar with Tetris games) find this game is both familiar to play, yet at the same time, presents basic life science concepts by repurposing contemporary game mechanisms and practices.

Multi-person game play-As we have indicated above, it is possible for an individual to play DQO without others. Beyond this, DQO also features a final level game module, DinoSphere, which is a multi-player or multi-character game module. In this module, player specify and configure a dinosaur of their choosing, using the resources and points they have earned from previous game play. DinoSphere features four ecological niches that serve as simulated physical world environments where different dinosaurs must survive or co-habitate. As players by this point have already learned about life science concepts like prey-predater relationships, then the quickly realize small predators (e.g., raptors) individually are not a threat to larger prey (stegosaurus), unless they can find other similar predators who can then collectively act to surround and overwhelm a larger prey. Figure 7 provides a view of a forest ecological niche within DinoSphere where one small raptor seeks to engage a larger stegosaurus as prey, but without success.

\section{Discussion and planned enhancements to DQO}

The first topic of discussion is whether the DQO games are fun to play. Since fun may be in the mind of the player, we can report results from sustained informal observations (along with a multitude of DSC administered survey questionnaires and interviews) that the quick and simple answer is: yes, they are fun to play, but each is fun in a different way. Second, do the players learn anything useful about scientific inquiry or life science? Again based on the same instrumentality for observation, the quick and simple answer is yes, though what is learned across DQO games is different. In both situations, DQO is a game environment played in an online, Web-based venue where other people may be involved in play, but their involvement may be centered around interaction at the human-computer interface or through in-game dinosaur characters foraging in a simulated ecological niche. So we expect that what's fun and what's learned will be different, but we continue to seek to understand how and why they are different.

First, reflecting the diversity of people (students, parents, and others) who visit DSC, we seek to provide multi-lingual game play user interfaces for DQO in lan- 
guages such as Spanish and Korean. Actually, we developed DQO with internationalization and localization as part of the design and implementation scheme, so provision of multi-lingual support is primary one of adding and replacing corresponding in-game textual content across languages.

Second, following from this, we seek to provide multi-national deployments for DQO to non-English speaking venues. Dinosaur themed interactive science exhibits are being developed in areas like Mexico, Latin America, and South Korea. Though DQO was designed with California Science Education Standards in mind, our correspondence with colleagues in those areas indicates such standards are acceptable as a starting point.

Third, we seek to expand the multi-player capabilities of the DQO DinoSphere to accommodate more MMOG services amd game play modes. Our intent is to add such capabilities to the highest levels of DQO-DinoSphere game play, so that existing game content and play experience will be minimally affected. The wisdom of this choice remains to be seen and realized.

Last, our original goals included making DQO a networked SLG environment that could be built from open source software, support open content artwork, and accommodate a controlled interface to the Web of open and current scientific research related to paleontology and paleobiology. None of this has been realized, and the technical choices that we have invested will limit what we can do to realize these goals. Nonetheless, they remain part of our long-term goal, and we look forward to opportunities that will enable to develop more collaborative SLGs in other scientific domains and for other public audiences who want informal science education experiences and resources.

\section{Conclusions}

In this paper, we described some of the concepts and results that arose from the development and deployment of a large-scale collaborative science learning game environment called DinoQuest Online. DQO provides an entertaining experience and approach to informal science education, as well as matching California/National Science Education Standards. As noted, games for helping school-age children to learn about science (or more specifically, life science) are not a focus of the global computer game industry, yet we believe it represents an important and under-served community of potential game players and others (parents, teachers) who want to informally collaborate in and around such a science learning game environment. Subsequently, we identified and examined different collaborative group forms that emerged to play DQO, as well as the affordances that help facilitate collaborative game play. Along the way we provided examples of the collaborative groups and game play from DQO. 
In closing, we welcome participants of the First IFIP Entertainment Computing Symposium to register and play one or more of the DinoQuest Online game modules found starting at http://www.DQOnline.org. DQO will provide experiences for collaborative games and game play which can last for 3-5 hours in total.

Acknowledgements: Development of DinoQuest Online was supported by the Discovery Science Center, its members, and many governmental and corporate sponsors. Recent R\&D projects at the UCGameLab are supported with contracts and grants from the Digital Industry Promotion Agency (DIP) in Daegu, South Korea, Intel Research, California Institute for Telecommunications and Information Technology (Calit2), and others. No endorsement implied. Some of the other participants involved in the development of DinoQuest Online include Alex Szeto (DQO game art and programming), Calvin Lee (DQO database programming), Janet Yamaguchi (California Science Education Standards, and Education Programs at DSC), and Celia Pearce (design contributions).

\section{References}

Allison-Bunnell, S. and Schaller, D.T. (2005). From the Physical to the Virtual: Bringing Free-Choice Science Education Online, in L. Tan and R. Subramanian (eds.), E-Learning and Virtual Science Centers, 163-189, Hershey, PA, IGI Global Publishing, 2005.

Bainbridge, W.S. (2007). The Scientific Research Potential of Virtual Worlds, Science, 317, 472-476.

Bogost, I. (2007). Persuasive Games: The Expressive Power of Videogames, MIT Press, Cambridge, MA.

Collabs, (2008). The Science of Collaboratories, http://www.scienceofcollaboratories.org/

DSC, (2008). Discovery Science Center, Santa Ana, CA. http://www/discoverycube.org

Gee, J.P. (2003). What Computer Games Have To Teach Us About Learning and Literacy, Palgrave Macmillan, New York.

Koster, R. (2004). A Theory of Fun for Game Design, Paraglyph Press.

LaFarge, A. and Nideffer, R. (2002). Shift-Ctrl: Computer, Games, and Art, Leonardo: A Journal of Art, Science, and Technology. MIT Press. 35(1), 5-13, Winter. 
Malone, T. and Lepper, M.R. (1987). Making Learning Fun: A taxonomy of intrinsic motivations for learning, in R.E. Snow and M.J. Farr, Aptitude, Learning, and Instruction III: Conative and Affective Process Analyses, 223-253, Erlbaum, Hillsdale, NJ.

Nardi, B. and Harris, J. (2006). Strangers and Friends: Collaborative Play in World of Warcraft, Proc. 2006 Conf. Computer Support Cooperative Work (CSCW'06), 149-158, Banff, Canada, ACM Press.

Nideffer, R. (2002). PROXY, The Whitney Museum Portal to Net Art, http://artport.whitney.org/exhibitions/biennial2002/nideffer.shtml.

Nideffer, R. (2006). unexceptional.net, http://transliteracies.english.ucsb.edu/ post/research-project/project-members/robert-nideffer

Nideffer, R. (2007). Game Engines as Embedded Systems, in V. Vesna (ed.), Database Aesthetics: Art in the Age of Information Overflow. University of Minnesota Press. Minneapolis, Minnesota.

Rieber, L.P. (2005). Multimedia Learning in Games, Simulations, and Microworlds, in R.E. Mayer (ed.), The Cambridge Handbook of Multimedia Learning, Cambridge University Press, Cambridge, UK.

Salen, K. and Zimmerman, E. (2003). Rules of Play: Game Design Fundamentals, MIT Press.

Scacchi, W. (2004). Free/Open Source Software Development Practices in the Computer Game Community, IEEE Software, 21(1), 59-67, January/February 2004.

Scacchi, W. and Adams, J. (2007). Recent Developments in Science Learning Games for Informal Science Education, presentation at Games, Learning, and Society: 3.0, Madison, WI. July.

Scacchi, W., Nideffer, R., and Adams, J. (2008). Collaborative Game Environments for Informal Science Education: DinoQuest and DinoQuest Online, 2008 Collaboration Technologies and Systems Symposium, Irvine, CA (submitted for publication).

Shaffer, D. W. (2006). How Computer Games Help Children Learn, Palgrave Macmillan, New York.

Teasley, S., \& Wolensky, S. (2001) Scientific collaborations at a distance. Science, 292, 2254-2255. 
UCGameLab, (2008). Game Culture and Technology Laboratory, University of California, Irvine, Irvine, CA. http://www.UCGameLab.net

WDIL (2008). Web Designs for Interactive Learning, http://www.wdil.org/

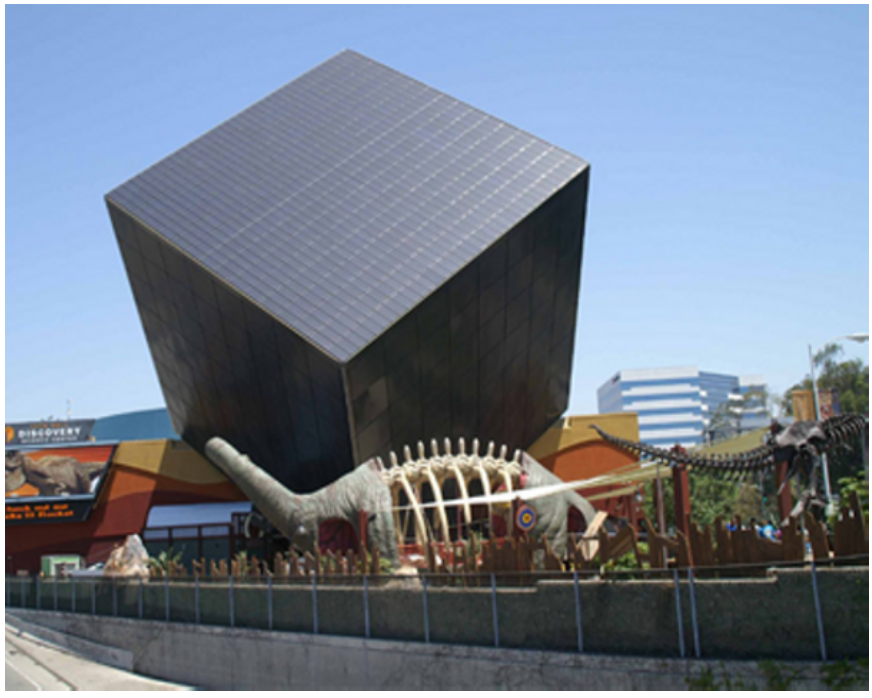

Figure 1. DinoQuest Physical Site.

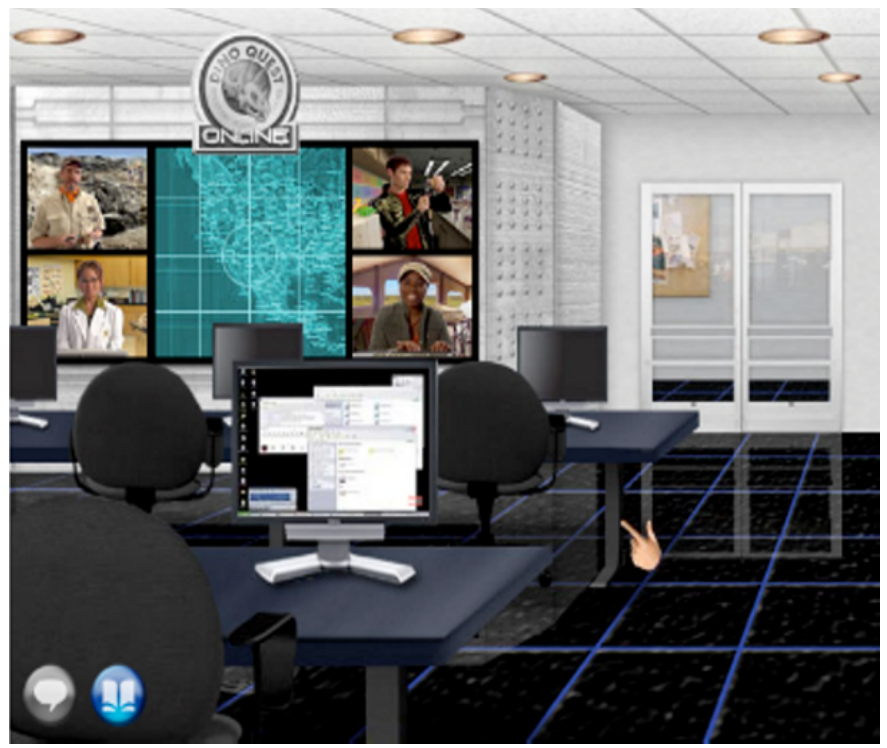

Figure 2. DinoQuest Online in-game home. 


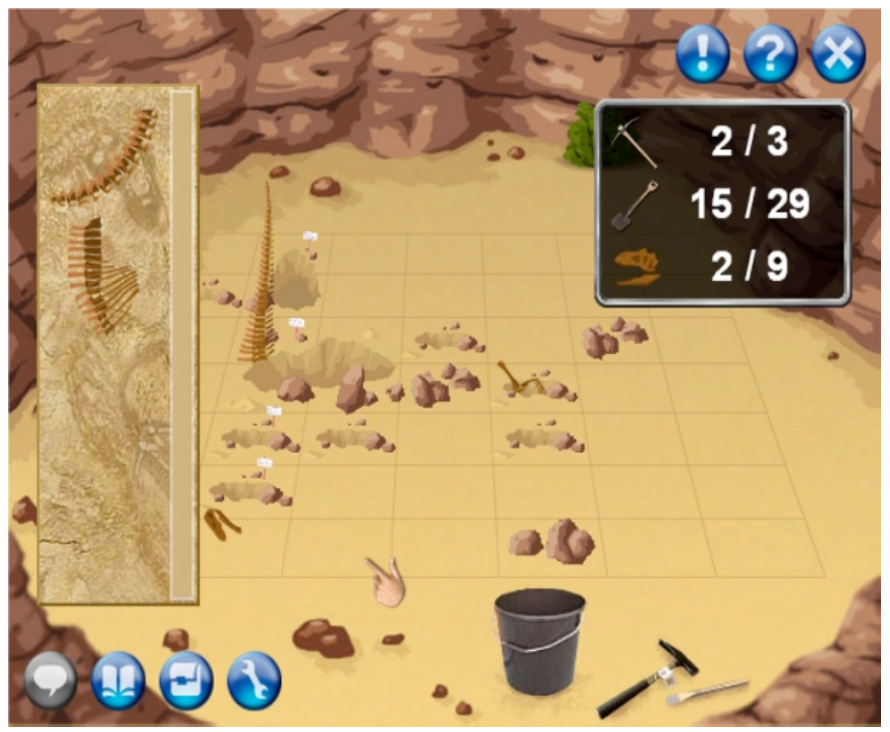

Figure 3. The Fossil Dig Pit game module showing different in-game user controls (hand, pick, shovel) and a dashboard indicating resource utilization (number of possible uses of the pick and shovel).

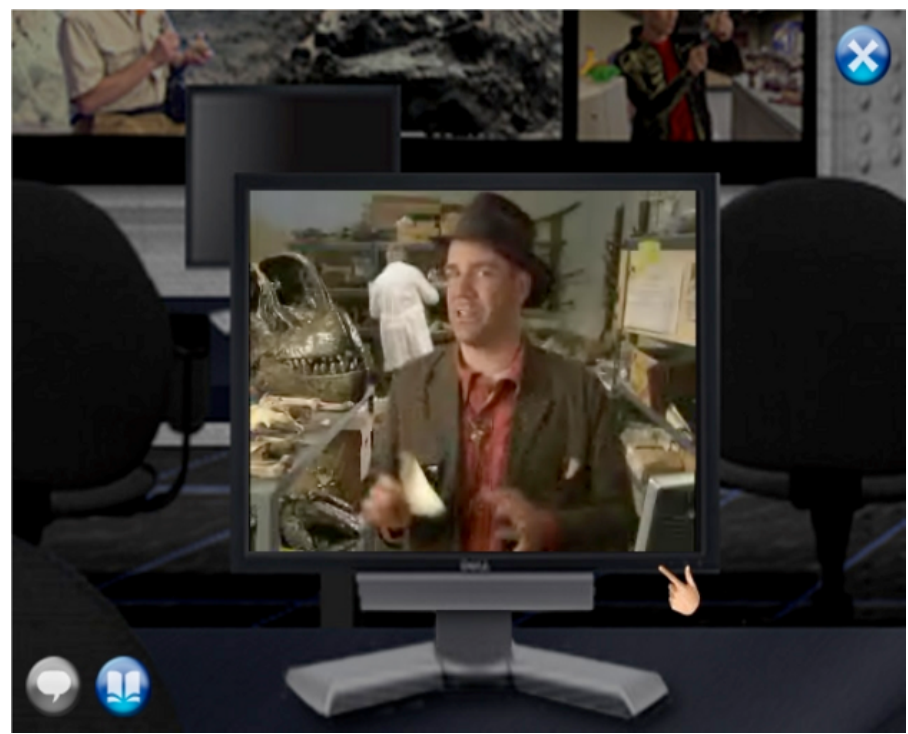

Figure 4. An in-game scene in DQO with an embedded video displaying featuring an in-character that introduces and explains DQO's goals and levels. 


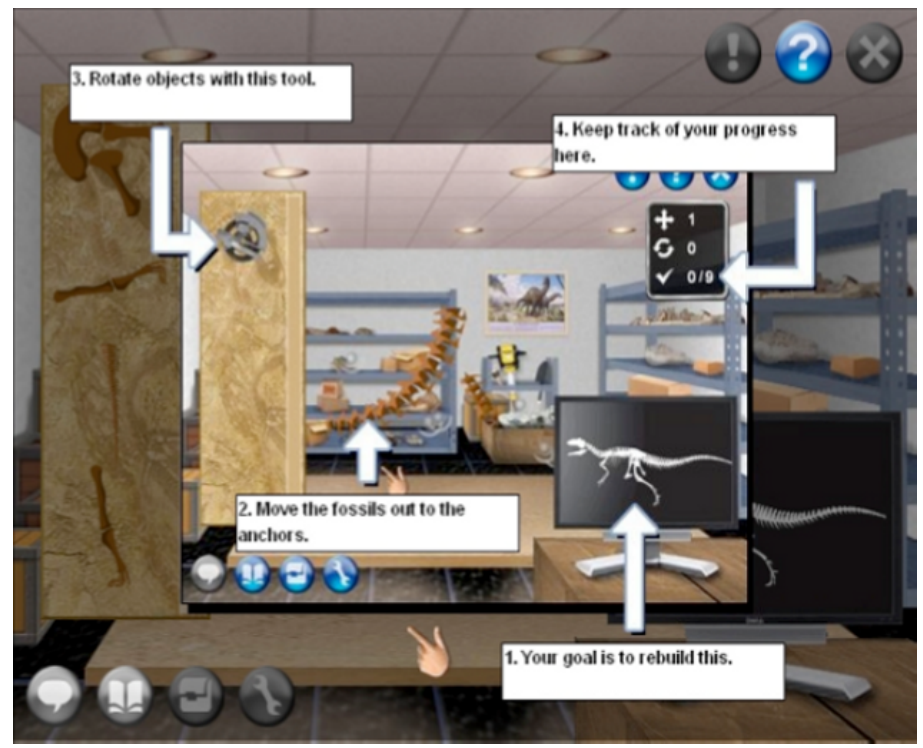

Figure 5. An in-game view of a teacher/parent tutorial explaining the goal and process for playing this DQO game module (reconstructing fossilized skeletal bones collected in the Fossil dig pit module).

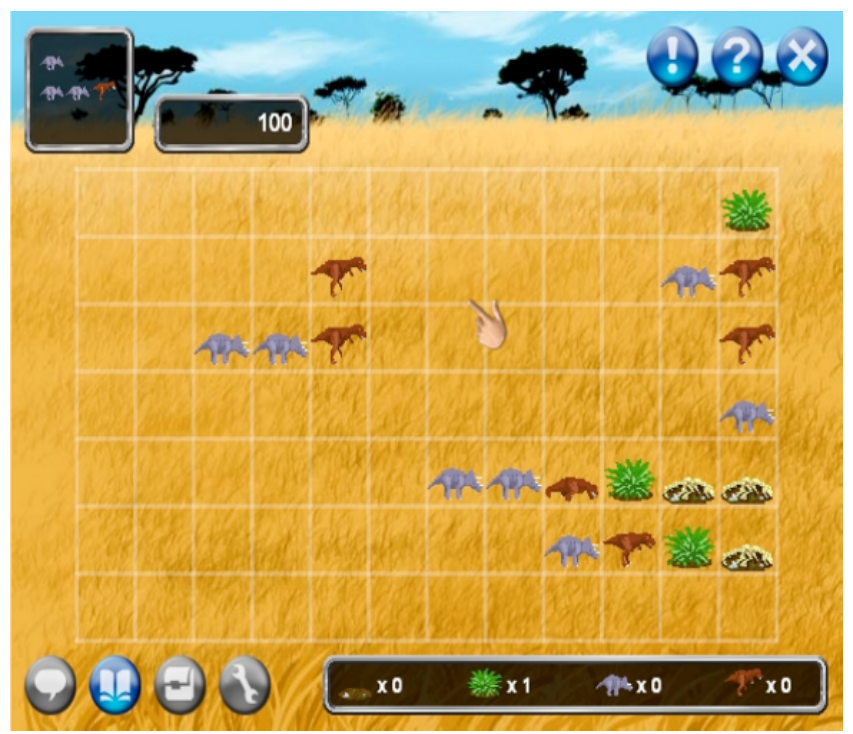

Figure 6. A Tetris-like game for matching ecological relationships like preypredator and food chains. 


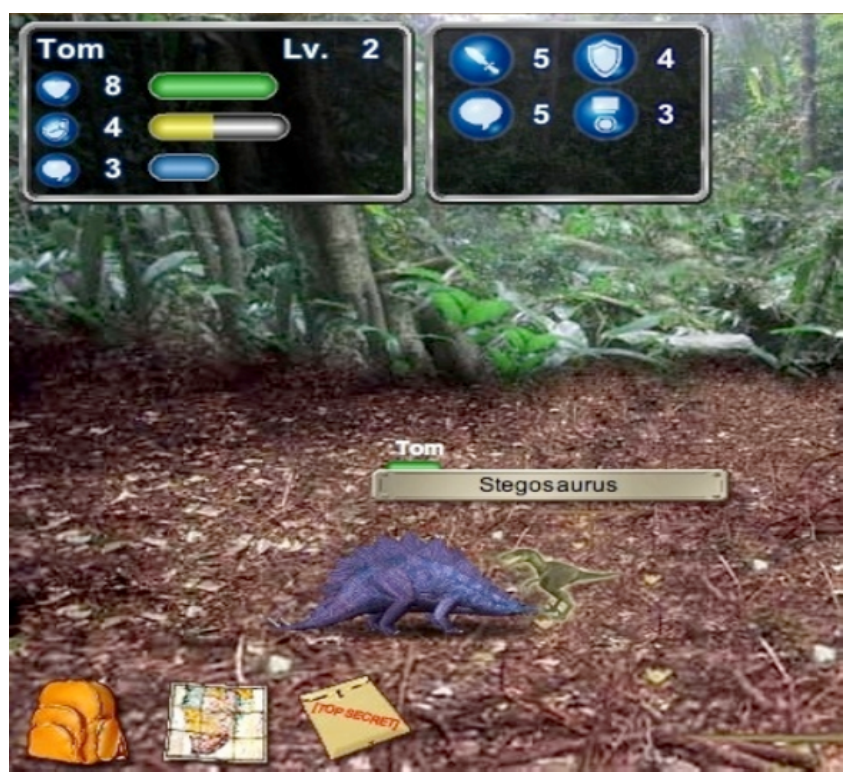

Figure 7. A scene from the DQO module, DinoSphere, where multiple players or in-game characters (e.g., stegosaurus and raptor) can interact in a simulated ecological niche to survive or thrive. The dashboard at the top displays the status of various resources controlled by the player Tom. 